\title{
Investigation of Microstrips with NiFe Magnetic Thin Film (II): Modelling
}

\author{
M. Vroubel, Y. Zhuang, B. Rejaei, J.N. Burghartz
}

Laboratory of Electronic Components, Technology \& Materials, Delft University of Technology, 2600 GB Delft, the Netherlands

In this paper, a model describing frequency dependent characteristics of a sandwiched microstrip with a ferromagnetic core, is developed. The approach is based on the assumption, that the characteristics of a sandwiched microstrip are mostly determined by a quasi-TEM mode. Frequency profiles of the inductance and resistance per unit length, attenuation, and wave-length are calculated. The increase of the inductance per unit length and the wavelength shortening are obtained due to the presence of a $\mathrm{NiFe}$ ferromagnetic core. The results of modeling are discussed and compared with experiments ${ }^{1}$.

Key words: RF, microstrip, soft-magnetic materials

\section{Introduction}

Microstrip transmission lines with hybrid magnetic/ dielectric cores have been investigated in several recent works ${ }^{2), 3)}$. Accurate modeling of such a device could help optimizing its structure. The theory of microwave propagation on multilayered structures has been discussed 4) -6). Nevertheless, references 4) and 5) did not include the influence of the finite width of the microstrip and reference 6) treated a multilayered ferromagnetic/oxide core as a uniform nonconductive magnetic layer.

In this work, the authors theoretically study the layered microstrip shown in Fig.1, and compare the results obtained from experimental data. The effects of the finite width of the microstrip and isolation layers are both taken into account. In particular, the influence of the finite width of the microstrip on the resonance characteristics of the structure is discussed.

The paper is organized as follows: Section 2 outlines the theoretical background and formulation of the model. Section 3 compares numerical results with experimental results, Section 4 provides a discussion and Section 5 contains concluding remarks.

\section{Theory}

It's assumed that the quasi-TEM approximation is valid for a magnetic microstrip ${ }^{7), 8)}$. This assumption allows to treat electrical and magnetic characteristics of the device independently, neglecting their mutual influence. It implies that the microstrip can be characterized by separately calculating the inductance $L$ and capacitance $C$ per unit length. Fig. 1 shows the cross-section of a microstrip with a hybrid ferromagnetic/dielectric core. It is assumed, that the easy axis of the ferromagnetic layer is parallel to the z-axis. The ferromagnetic layer is characterized by the permeability tensor $\ddot{\mu}{ }^{9)}$,

$$
\vec{\mu}=\left(\begin{array}{ccc}
\mu & \mathrm{j} \mu_{\alpha} & 0 \\
-\mathrm{j} \mu_{\alpha} & \mu & 0 \\
0 & 0 & 1
\end{array}\right),
$$

with

$$
\begin{aligned}
& \mu=1+\frac{\omega_{0} \omega_{\mathrm{a}}}{\omega_{\mathrm{a}}^{2}+1 / \mathrm{T}^{2}-\omega^{2}+2 j \omega / \mathrm{T}}, \\
& \mu_{\alpha}=\frac{\omega_{0}(\omega-\mathrm{j} / \mathrm{T})}{\omega_{\mathrm{a}}^{2}+1 / \mathrm{T}^{2}-\omega^{2}+2 j \omega / \mathrm{T}},
\end{aligned}
$$

where $T$ is a relaxation time corresponding to the phenomenological damping parameter in Bloch form ${ }^{10}$ ), 11), $\omega_{0}=\gamma M_{0}, \omega_{a}=\gamma H_{a}, \gamma$ is the gyromagnetic ratio, $H_{a}$ is the anisotropy field, and $M_{0}$ is the saturation magnetization. In order to determine the inductance per unit length, we assume, that a current $J=J_{\mathrm{z}}(\mathrm{x})$ is flowing in the microstrip. Due to the uniformity of the system in the z-direction, only the components $E_{\mathrm{z}}, H_{\mathrm{x}}$ and $H_{\mathrm{y}}$ are present. Maxwell's equations can then be reduced to the Helmholtz's equation, which, in each layer can be written as:

$$
\frac{\partial^{2} E_{z}}{\partial x^{2}}+\frac{\partial^{2} E_{z}}{\partial y^{2}}+\omega^{2} \varepsilon_{0} \mu_{0} \tilde{\varepsilon}_{i} \mu_{e i} E_{z}=0,
$$

where $\varepsilon_{0}$ and $\mu_{0}$ are, respectively, the permittivity and permeability of free space, $\tilde{\varepsilon}_{i}=\left(\varepsilon_{i}-j \sigma_{i} / 4 \pi \varepsilon_{0} \omega\right)$, with $\varepsilon_{i}$, $\sigma_{i}$ the relative permittivity, and conductivity of the $\mathrm{i}$-th layer; $\mu_{\mathrm{ei}}$ equals to 1 in all layers except the ferromagnetic core $\left(\mu_{\mathrm{ei}}=1\right.$ for $\left.\mathrm{i}=1,2,4,5,6\right)$, and corresponds to the effective transverse permeability $\mu_{\mathrm{e}}$ inside the ferromagnetic layer $\left.\left(\mu_{\mathrm{ei}}=\mu_{\mathrm{e}} \text { for } \mathrm{i}=3\right)^{9}\right)^{9}$, 13).

$$
\begin{aligned}
& \mu_{e}=\left(\mu^{2}-\mu_{\alpha}{ }^{2}\right) / \mu=1+\frac{\omega_{0}\left(\omega_{0}+\omega_{a}\right)}{\omega_{R}^{2}-\omega^{2}+2 j \omega / T} \\
& \omega_{R}^{2}=\omega_{0}\left(\omega_{\mathrm{a}}+\omega_{0}\right)+1 / \mathrm{T}^{2} .
\end{aligned}
$$

In the spectral domain, Eq. (3) is transformed to:

$$
\frac{\partial^{2} \tilde{E}_{z}}{\partial y^{2}}-\left(q^{2}-\omega^{2} \varepsilon_{0} \mu_{0} \tilde{\varepsilon}_{i} \mu_{e i}\right) \tilde{E}_{z}=0
$$

where $\tilde{E}_{z}(q, y)$ is the Fourier transform of $E_{\mathrm{z}}(x, y)$ :

$$
\tilde{E}_{z}(q, y)=\int_{-\infty}^{\infty} e^{-j q x} E_{z}(x, y) d x
$$

The solutions of equation (5) in regions 1-6 (Fig.1) are expressed as: 


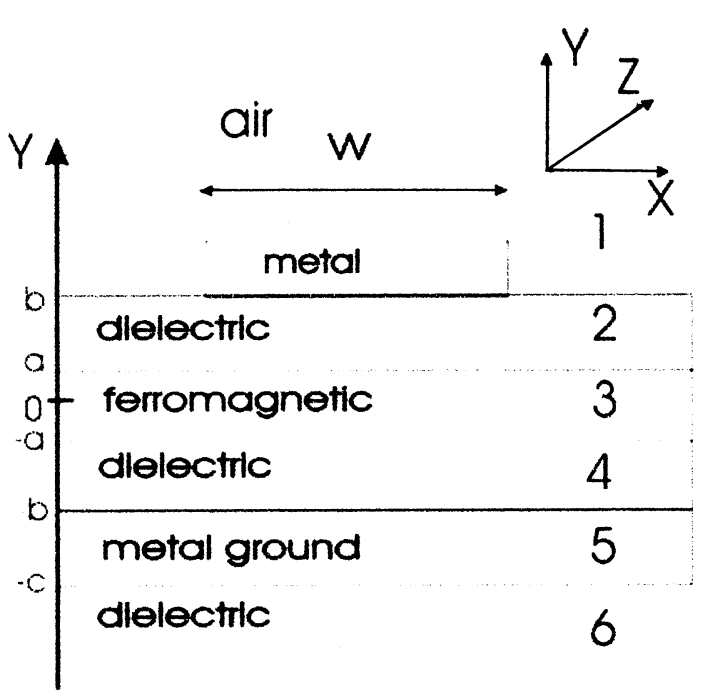

Fig.1 Schematic structure of a microstrip. The metal strip line is oriented along the $\mathrm{z}$-direction; the thickness of a ferromagnetic layer $=0.5 \mu \mathrm{m}$; the distance between the top strip and metal ground $2 b=2.5 \mu \mathrm{m}$; the thickness of the metal ground $c-b=2 \mu \mathrm{m}$; the thickness of the top strip equals to $3 \mu \mathrm{m}$, the width of the top strip $W=50 \mu \mathrm{m}$. Metal strip and ground layer are aluminum with conductivity $\sigma_{\mathrm{m}}=33 \times 10^{6} \mathrm{~S} / \mathrm{m}$; the conductivity of a $\mathrm{Ni}_{80} \mathrm{Fe}_{20}$ ferromagnetic core is $6.25 \times 10^{6} \mathrm{~S} / \mathrm{m}$.

$$
\begin{array}{ll}
\tilde{E}_{z}=A_{i} e^{-k_{i} y} & i=1 \\
\tilde{E}_{z}=A_{i} e^{k_{i} y} & i=6 \quad k_{i}^{2}=q^{2}-\varepsilon_{0} \mu_{0} \tilde{\varepsilon}_{i} \mu_{e i} \omega^{2},(7) \\
\tilde{E}_{z}=A_{i} e^{k_{i} y}+B_{i} e^{-k_{i} y} & i=2,3,4,5
\end{array}
$$

where $A_{i}, B_{i}$ are arbitrary constants. Applying the usual boundary conditions at the interfaces:

$\mathrm{E}_{\mathrm{zi}}=\mathrm{E}_{\mathrm{zi}+1}$

$\mathrm{H}_{\mathrm{xi}+1}-\mathrm{H}_{\mathrm{xi}}=-\mathrm{J}_{\mathrm{z}}(\mathrm{x})$

and taking into account, that ${ }^{9), 13)}$

$$
H_{x}=\frac{1}{\mu_{e}}\left(B_{x}-j \frac{\mu_{\alpha}}{\mu} B_{y}\right)=-\frac{j}{\mu_{e} \omega}\left(\frac{\partial E_{z}}{\partial y}+j \frac{\mu_{\alpha}}{\mu} \frac{\partial E_{z}}{\partial x}\right)
$$

we can find coefficients $\mathrm{A}_{\mathrm{i}}, \mathrm{B}_{\mathrm{i}}$ and compute $\tilde{E}_{z}$ everywhere in the space. But since we are interested in the microstrip structure, we just evaluate $\tilde{\mathrm{E}}_{\mathrm{z}}$ on the surface $y$ $=b$. Taking into account, that $\mu_{\mathrm{e}}=1$ and $\mu_{\alpha}=0$ in all layers except the ferromagnetic core and assuming that

$$
k_{1} \approx k_{2}=k_{4} \approx q \quad\left(q^{2} \sim 1 / W^{2}>\omega^{2} \varepsilon_{0} \mu_{0} \varepsilon_{i}\right)
$$

in the frequency range of interest $(<10 \mathrm{GHz})$ for the microstrip width $W=50 \mu \mathrm{m}$, we obtain :

$$
\tilde{E}_{y}=j \tilde{J}_{z} \omega Y
$$

$$
\begin{aligned}
& Y=\frac{1}{2 q}(1+ \\
& \mathrm{e}^{-2 \mathrm{q}(\mathrm{b}-\mathrm{a})} \frac{\left(-\frac{\mathrm{k}_{\mathrm{F}}}{\mu_{\mathrm{e}}}-\frac{\mathrm{q}}{\mu_{\mathrm{e} \alpha}}+\mathrm{q}\right) \mathrm{e}^{2 \mathrm{k}_{\mathrm{F}} \mathrm{a}}+X\left(\frac{\mathrm{k}_{\mathrm{F}}}{\mu_{\mathrm{e}}}-\frac{\mathrm{q}}{\mu_{\mathrm{e} \alpha}}+\mathrm{q}\right) \mathrm{e}^{-2 \mathrm{k}_{\mathrm{F}} \mathrm{a}}}{\left.\left(\frac{\mathrm{k}_{\mathrm{F}}}{\mu_{\mathrm{e}}}+\frac{\mathrm{q}}{\mu_{e \alpha}}+\mathrm{q}\right) \mathrm{e}^{2 \mathrm{k}_{\mathrm{F}} \mathrm{a}}-X\left(\frac{\mathrm{k}_{\mathrm{F}}}{\mu_{\mathrm{e}}}-\frac{\mathrm{q}}{\mu_{e \alpha}}-\mathrm{q}\right) \mathrm{e}^{-2 \mathrm{k}_{\mathrm{F}} \mathrm{a}}\right)} \\
& X=\frac{\left(\frac{k_{F}}{\mu_{e}}+\frac{q}{\mu_{e \alpha}}-q\right) e^{q(b-a)}+Z\left(\frac{k_{F}}{\mu_{e}}+\frac{q}{\mu_{e \alpha}}+q\right) e^{-q(b-a)}}{\left(\frac{k_{F}}{\mu_{e}}-\frac{q}{\mu_{e \alpha}}+q\right) e^{q(b-a)}+Z\left(\frac{k_{F}}{\mu_{e}}-\frac{q}{\mu_{e \alpha}}-q\right) e^{-q(b-a)}} \\
& \mathrm{Z}=\frac{\left(\mathrm{q}^{2}-\mathrm{k}_{\mathrm{m}}{ }^{2}\right)\left(1-\mathrm{e}^{-2(\mathrm{c}-\mathrm{b}) \mathrm{k}_{\mathrm{m}}}\right)}{\left(\mathrm{q}+\mathrm{k}_{\mathrm{m}}\right)^{2}-\mathrm{e}^{-2(\mathrm{c}-\mathrm{b}) \mathrm{k}_{\mathrm{m}}}\left(\mathrm{q}-\mathrm{k}_{\mathrm{m}}\right)^{2}}
\end{aligned}
$$

where $k_{m}$ and $k_{F}$ are determined by (7) and correspond to the metal ground and ferromagnetic layer, respectively, $\mu_{e}$ is an effective transverse permeability of the ferromagnetic layer (4),) and $\mu_{\mathrm{e} \alpha}=\left(\mu^{2}-\mu_{\mathrm{a}}^{2}\right) / \mu_{\mathrm{a}}$ with $\mu$ and $\mu_{\mathrm{a}}$ being the matrix components (2). By an inverse Fourier transform we have:

$E_{z}(x, y)=\frac{1}{2 \pi} \int_{-\infty}^{\infty} e^{j q x} \tilde{E}_{z}(q, y) d q$,

where $E_{\mathrm{z}}$ is the field of a point source at $x=0, y=b$. For a strip on the plane $y=b$ with a current density $J_{z}(x)$ we have the electric field:

$E_{S z}(x, y=b)=\int_{x_{1}=-w / 2}^{x_{2}=w / 2} E_{z}\left(x-x^{\prime}, y=b\right) J_{z}\left(x^{\prime}\right) d x^{\prime}$

The impedance of the structure can be calculated through the complex power:

$$
\mathrm{Z}=\frac{\mathrm{P}}{\mathrm{I} \cdot \mathrm{I}^{*}}=\frac{\int_{\mathrm{x}_{1}=-\mathrm{W} / 2}^{\mathrm{x}_{2}=\mathrm{W} / 2} \mathrm{E}_{\mathrm{Sz}}(\mathrm{x}) \mathrm{J}(\mathrm{x})^{*} \mathrm{dx}}{\mathrm{I} \cdot \mathrm{I}^{*}},
$$

where $*$ corresponds to the complex conjugation and

$$
I=\int_{-W / 2}^{W / 2} J_{z}(x) d x
$$

is the total current flowing through the microstrip. Assuming that the current distribution on the strip is given by $J_{z}(x)=A_{0} /\left(1-(2 x / W)^{2}\right)^{1 / 27)}$, from (10)-(14) we have:

$Z=\frac{j \omega}{\pi} \int_{-\infty}^{\infty} d q Y(q, y=b) J_{0}^{2}(q W / 2)$,

where $J_{0}$ is the Bessel function of the zeroth-order. If the current distribution $J_{z}(x)$ is taken as uniform, the result (15) reduces to: 
$Z=\frac{j \omega}{\pi} \int_{-\infty}^{\infty} d q Y(q, y=b)\left(\frac{\sin (q W / 2)}{q W / 2}\right)^{2}$

which, from our estimations, gives sufficient accuracy for $W / b>>1$ and considerably reduces the time of calculation. The inductance and resistance per unit length can be calculated as:

$\mathrm{L}=\frac{1}{\omega} \operatorname{Im}(Z), \quad \mathrm{R}=\operatorname{Re}(\mathrm{Z})+\mathrm{R}_{1}$,

where $R_{l}$ is the resistance per unit length of the top microstrip.

So far we calculated the inductance $L$ per unit length. The capacitance $C$ per unit length can be calculated by assuming the microstrip to be held at a constant potential with respect to the microstrip and computing the resulting charge density on the microstrip ${ }^{7)}$. Instead, however, we neglect the fringe fields and approximated $C$ by $C=C_{1} C_{2} /\left(C_{1}+C_{2}\right), C_{\mathrm{i}}=\varepsilon_{\mathrm{ox}} W_{\mathrm{i}} / d_{\mathrm{i}}$, where $\varepsilon_{\mathrm{ox}}$ is the dielectric constant of the oxide, $W_{1}=W ; \quad W_{2}=200 \mu \mathrm{m}$ is the width of the magnetic core; $d_{1}=d_{2}=b-a$ are the distances between the microstrip and the conducting ferromagnetic core and between the ferromagnetic core and the ground respectively. The propagation constant for known $L, R$ and $C$, neglecting the conductance $G$, is determined by :

$\gamma=\sqrt{(R+j \omega L) j \omega C}$.

The real part of $\gamma$ is the attenuation constant, and the imaginary part of $\gamma$ is inversly proportional to the propagation wavelength in the microstrip.

\section{Results}

The effective transverse permeability, which was taken for calculations in this paper, is given in Fig 2. The transverse effective permeability was calculated using Eq.(4) with $M_{0}=0.35 \mathrm{~T}$ and $H_{\mathrm{a}}=38 \mathrm{Oe}$ obtained from B-H loop measurement. The high value of the anisotropy field can be explained by the shape anisotropy effect ${ }^{12)}$ for the ferromagnetic core with $L_{\mathrm{m}}>>W_{\mathrm{m}}, W_{\mathrm{m}} / T_{\mathrm{m}}=400$, where $W_{\mathrm{m}}$, $T_{\mathrm{m}}$ and $L_{\mathrm{m}}$ are the sizes of the ferromagnetic core in the $x$, $y$ and $z$-directions, respectively. The damping parameter $\mathrm{T}=1.75 \mathrm{~ns}$ was estimated as a fitting parameter by matching calculated and measured results for a number of structures. The structural characteristics of the microstrip are given in the caption to Fig.1

The calculated and measured inductance and resistance as function of frequency for the microsrip with and without a $\mathrm{NiFe}$ ferromagnetic core are shown in Fig. 3 and 4 respectively. The inductance of the microstrip with a ferromagnetic core (Strip1, Fig.3) shows the enhancement over the microstrip without a magnetic core (Strip2, Fig.4). The inductance of Strip2 falls sharply for frequencies below $1 \mathrm{GHz}$ (Fig.4) due to increasing negative mutual inductance arising from current induced in the ground plane. The amplitude of the mutual inductance reaches a maximum and the inductance stays almost constant above $1 \mathrm{GHz}$. From our simulations, this effect is practically negligible for Strip1 in the frequency range between $0.1-1 \mathrm{GHz}$, because the presence of a ferromagnetic core compensates the already mentioned negative mutual inductance.

Comparison of the inductance of Strip1 (Fig.3) with the permeability profile of Fig.2 shows two important features. Firstly, below the ferromagnetic resonance, where $\mu_{\mathrm{e}}^{\prime}>0$ and $\mu_{\mathrm{e}}^{\prime}>\mu_{\mathrm{e}}^{\prime \prime}$ (Fig.2), the inductance undergoes a slow drop. Around the FMR frequency $\omega_{R}$, the frequency profile of the permeability undergoes a sharp changes due to FMR (Fig.2), yielding a strong decrease in inductance (Fig.3). However, the frequency $\omega_{\mathrm{LR}}$ at which $L=0$, is higher than the FMR frequency $\omega_{\mathrm{R}}$. The discussion of both these effects will be given in the next section.

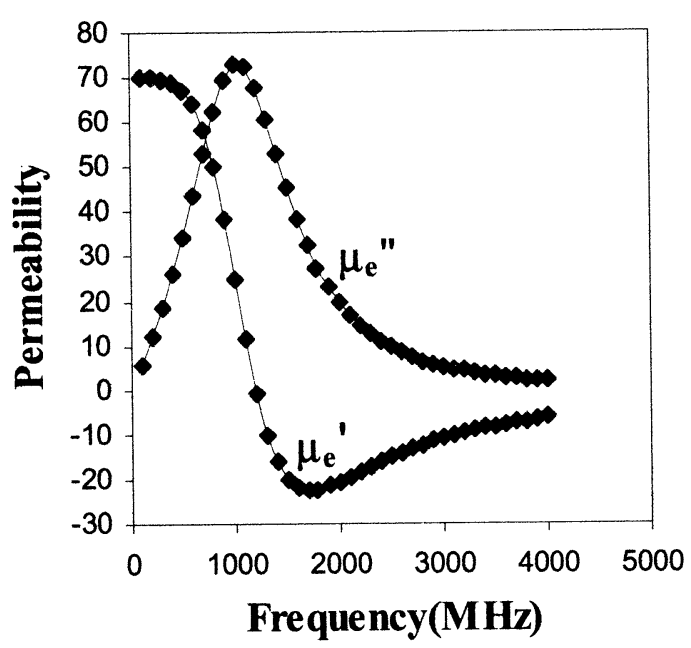

Fig. 2 Calculated permeability spectrum for the effective transverse permeability $\mu_{\mathrm{e}}=\mu_{\mathrm{e}}^{\prime}-\mathrm{j} \mu_{\mathrm{e}}^{\prime \prime}$ (2), (4); for $M_{0}=0.35 \mathrm{~T}$, $H_{\mathrm{a}}=380 \mathrm{e}, T=1.75 \mathrm{~ns}$.

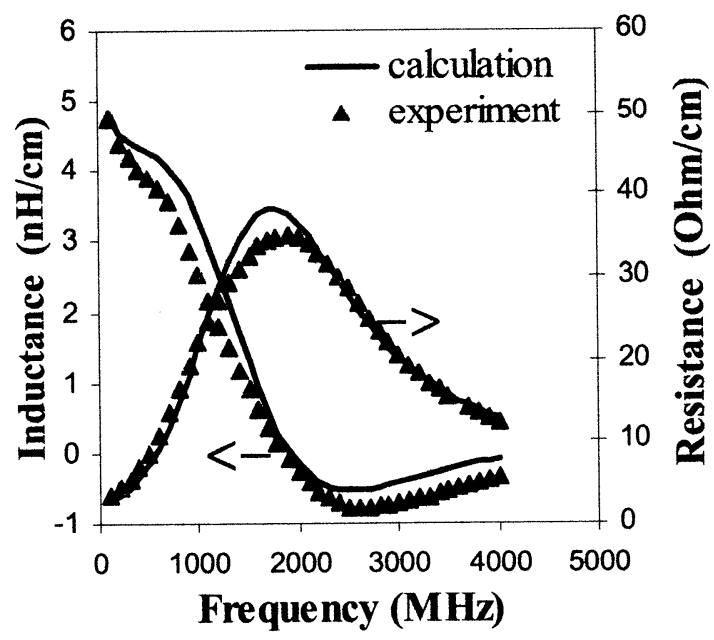

Fig. 3 Measured and calculated inductance $L$ and resistance $R$ per unit length as a function of frequency for microstrip with a ferromagnetic core (Strip1). 


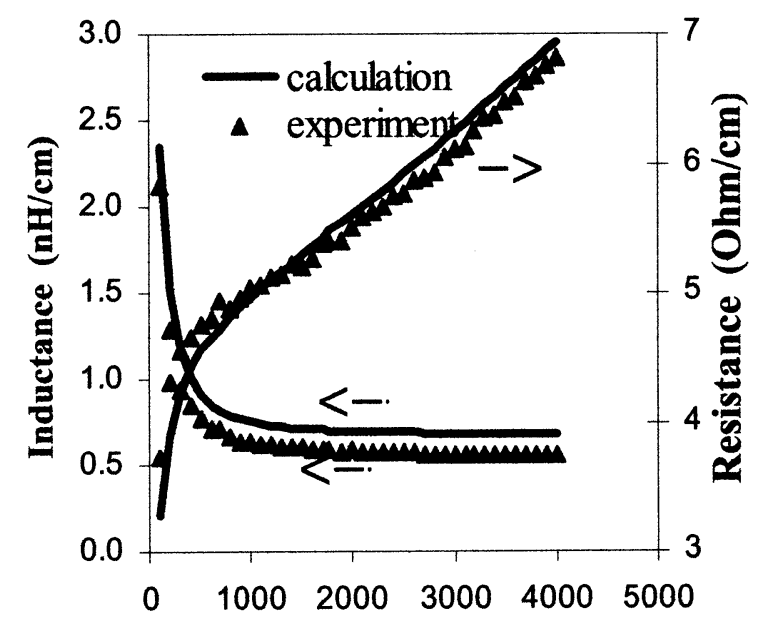

Frequency (MHz)

Fig. 4 Measured and calculated inductance $L$ and resistance $R$ per unit length as a function of frequency for a microstrip without a ferromagnetic core (Strip2).

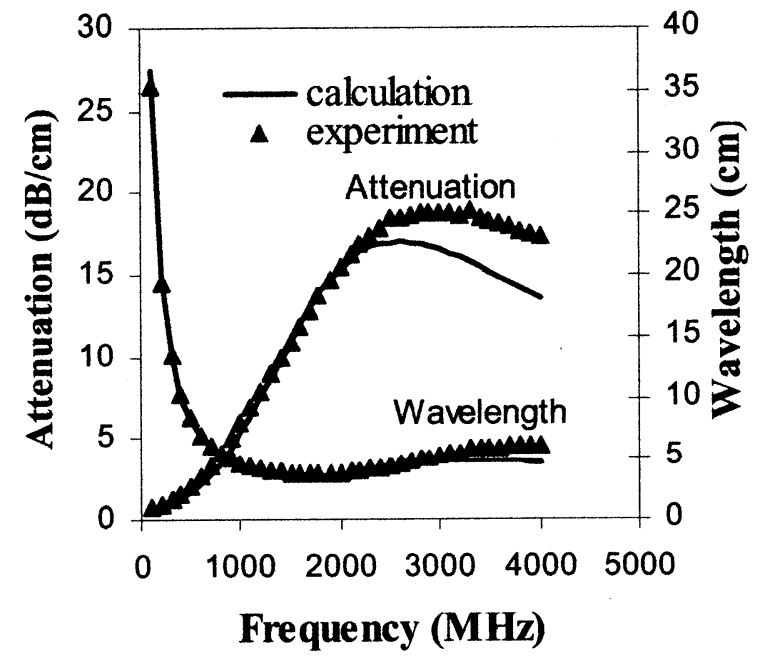

Fig. 5 Measured and calculated attenuation and wavelength per unit length as a function of frequency for microstrip with a ferromagnetic core.

The insertion of a magnetic core enhances the losses of the structure. For the microstrip with a ferromagnetic core the resistance $R$ contains (i) ohmic conductor losses in the microstrip and eddy current losses in the conducting ground; (ii) eddy-current and magnetic absorption losses in the ferromagnetic core. The first component should increase monotonically with increasing frequency. The second component has the resonance behavior with a maximum beyond the FMR frequency (Fig.3). From our simulations, in the frequency range between 0.5 and
$3.5 \mathrm{GHz}$, the ferromagnetic absorption represented by $\mu_{\mathrm{e}}^{\prime \prime}$, makes the major part of losses. The maximum of $\mathrm{R}$ is shifted beyond the FMR frequency (similar to $\omega_{\mathrm{LR}}$ for the inductance) as will be explained in Section 4.

The attenuation constant and the wavelength (Fig.5 and 6) were calculated using Eq. (18). The shortening of the propagation wavelength by the insertion of the ferromagnetic core is a result of the increase in not only the inductance, but also the capacitance per unit length (see Eq. (18)). The capacitance of the microstrip with a magnetic core is approximately twice higher than that of the microstrip without a magnetic core, because of the high conductivity of the core. The resistance $R$ also contributes to the wavelength reduction, especially at small values of inductance $L$ (at frequencies around and above $\left.\omega_{\mathrm{RL}}\right)$. The wavelength for the strip with a ferromagnetic core decreases with frequency, becomes almost constant above FMR frequency, and increases slowly above $1.5 \mathrm{GHz}$ (Fig.5). The wavelength of the conventional microstrip is a decreasing function through the whole frequency region (Fig.6).

Above the frequency $\omega_{\mathrm{LR}} \approx 1.9 \mathrm{GHz}$, the inductance of the microstrip with a ferromagnetic core is negative, hence there is no wave propagation above $\omega_{\mathrm{LR}}$, and the real part of $\gamma$ characterizes not the wavelength, but $R-C$ effects in this frequency region. The effect, however, is not attractive from the point of view of RF device design.

The calculated and measured wavelength reduction factor defined as the ratio of the wavelength of the microstrip with and without magnetic core are shown in Fig.7. The wavelength reduction is maximum around $1 \mathrm{GHz}$, the FMR frequency and, as already mentioned, is useless after $\omega_{\mathrm{LR}}$.

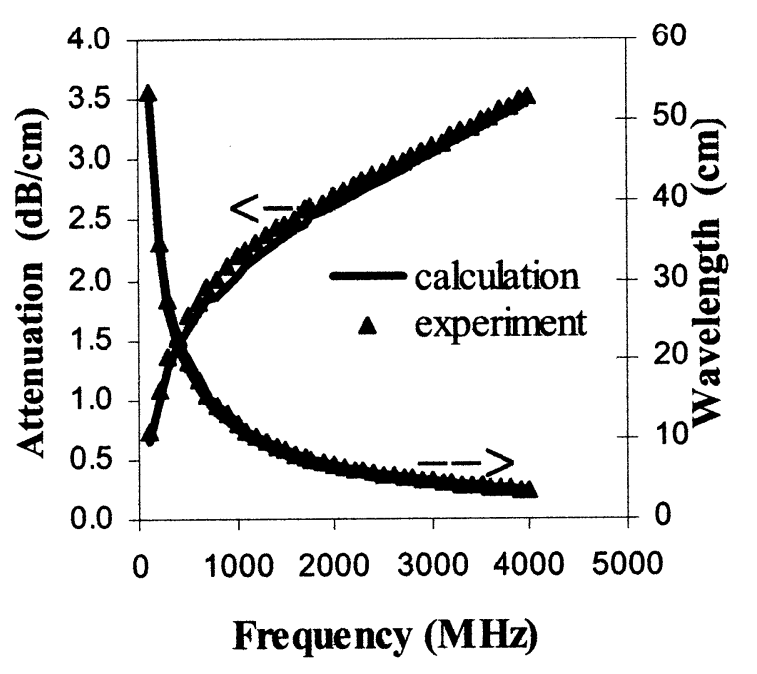

Fig. 6 Measured and calculated attenuation and wavelength per unit length as a function of frequency for microstrip without a magnetic core. 


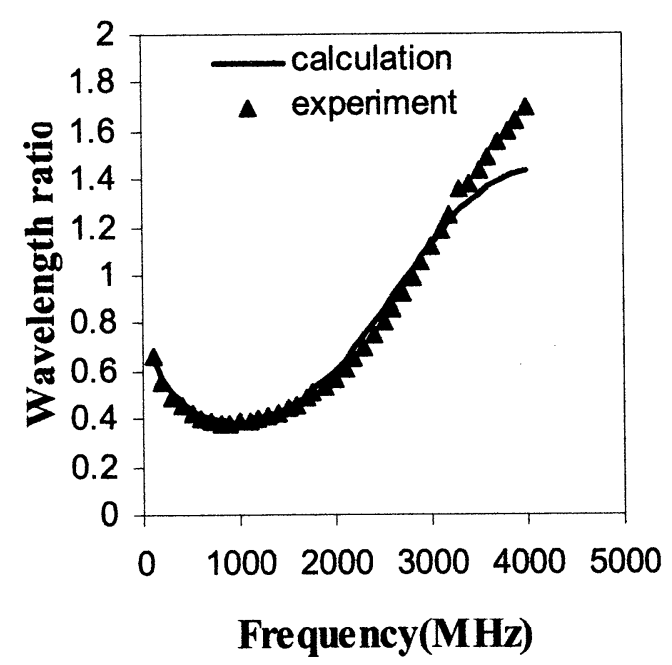

Fig. 7 Measured and calculated wavelength ratio for microstrip with and without magnetic core.

\section{Discussion}

In what follows, we argue, that the shift of the frequency $\omega_{\mathrm{LR}}, L\left(\omega_{\mathrm{LR}}\right)=0$, beyond the FMR frequency can be attributed to the finiteness of the width of the microstrip, whereas the slow drop of the inductance below the FMR is a result of conductivity of the ferromagnetic core. To understand these effects, let us consider Eqs.(10) where we make several assumptions for further simplification. We assume, that the ground plane is a perfect conductor. As a result, $Z=-1$ in Eq. (10). For the moment, consider the structure without isolation layers. The last assumption along with $Z=-1$ leads to $X=-1$. Then $Y$ reduces to:

$$
\mathrm{Y}=\frac{2 \mathrm{a} \mu_{\mathrm{e}} \tanh \left(2 \mathrm{k}_{\mathrm{F}} \mathrm{a}\right) /\left(2 \mathrm{k}_{\mathrm{F}} \mathrm{a}\right)}{1+2 \mathrm{a}\left(\mathrm{q} / \mu_{\mathrm{e} \alpha}+\mathrm{q}\right) \mu_{\mathrm{e}} \tanh \left(2 \mathrm{k}_{\mathrm{F}} \mathrm{a}\right) /\left(2 \mathrm{k}_{\mathrm{F}} \mathrm{a}\right)}
$$

where $\mu_{\mathrm{ea}}(\omega)$ is a decreasing function with $\mu_{\mathrm{e} \alpha}(0) \rightarrow \infty$ and $\mu_{e \alpha}\left(\omega_{R}\right) \sim\left(M_{0} / H_{a}\right)^{1 / 2}>1^{9)}$, Then, for $\omega \leq \omega_{\mathrm{R}}$, we can write: $2 a\left(q / \mu_{e \alpha}+q\right) \approx 2 a q$. Let us consider a nonconductive magnetic core. For such a core, $k_{F}=\left(q^{2}-\omega^{2} \varepsilon_{0} \mu_{0} \varepsilon_{F} \mu_{e}\right)^{1 / 2}$. At frequency below $10 \mathrm{GHz}$, for $q \sim 1 / W, W=50 \mu \mathrm{m}, \quad a=0.5 \mu \mathrm{m}$, the condition $2 k_{F} a<<1$ leads to the estimation $\mu_{\mathrm{e}}<10^{6}$, which is always true for any realistic material. Eq.(19) can be further reduced to: $Y=2 a \mu_{e} /\left(1+2 a q \mu_{e}\right)$. The real part of $Y$ is given by

$$
\operatorname{Re}(\mathrm{Y})=\frac{2 \mathrm{a}\left[\mu_{\mathrm{e}}^{\prime}+2 \mathrm{aq}\left(\mu_{\mathrm{e}}^{\prime 2}+\mu_{\mathrm{e}}^{\prime \prime 2}\right)\right]}{\left(1+2 \mathrm{aq} \mu_{\mathrm{e}}^{\prime}\right)^{2}+\left(2 \mathrm{aq} \mu_{\mathrm{e}}^{\prime \prime}\right)^{2}}
$$

which is proportional to the inductance per unit length (15)-(17). Here $\mu_{\mathrm{e}}^{\prime}$ and $-\mu_{\mathrm{e}}^{\prime \prime}$ are the real and imaginary parts of the effective permeability, respectively (Fig.2).
If $q \rightarrow 0$ (the width of the microstrip goes to infinity), Eq.(20) will include only the real part of the effective transverse permeability. But for finite widths of microstrips $(q>0)$, it follows from (20) that the increase of both, $\mu_{\mathrm{e}}^{\prime}$ and $\mu_{\mathrm{e}}^{\prime \prime}$ leads to the increase of $\operatorname{Re}(Y)$ and consequently $L$, as long as $\mu_{\mathrm{e}}^{\prime}>0$. The influence of $\mu_{\mathrm{e}}^{\prime \prime}$ on $\operatorname{Re}(Y)$ is stronger for higher values of $q$, i.e. smaller values of $W$. Nevertheless, as a whole, the effect of $\mu_{\mathrm{e}}^{\prime \prime}$ is not positive, because it increases $\operatorname{Im}(Y)$ and, consequently, the resistance per unit length:

$\operatorname{Im}(Y)=\frac{2 a \mu_{e}^{\prime \prime}}{\left(1+2 a q \mu_{e}^{\prime}\right)^{2}+\left(2 a q \mu_{e}^{\prime \prime}\right)^{2}}$.

It's easy to see from Eq.(20), that $\operatorname{Re}(Y)$ and $L$ will equal to 0 , when

$\mu_{\mathrm{e}}^{\prime}+2 \mathrm{aq}\left(\mu_{\mathrm{e}}^{\prime 2}+\mu_{\mathrm{e}}^{\prime \prime 2}\right)=0$.

If $q \rightarrow 0$, Eq. (22) reduces to the conventional condition $\mu_{\mathrm{e}}^{\prime}=0$. But for a finite-width microstrip, the inductance per unit length will cross zero when the real part of the effective transverse permeability is not zero, but negative, which happens at a frequency $\omega_{L R}>\omega_{R}$, increasing with the increase of $q \sim 1 / W$.

A similar effect takes place for the resistance. From Eq.(21), $\operatorname{Im}(Y)$ and consequently the resistance per unit length, depend on both, $\mu_{\mathrm{e}}^{\prime \prime}$ and $\mu_{\mathrm{e}}^{\prime}$. Taking into account, that $\mu_{\mathrm{e}}^{\prime}<0$ above the FMR frequency, from (21) one can see, that $\operatorname{Im}(Y(\omega))$ can be still an increasing function of frequency even after $\mu_{\mathrm{e}}^{\prime \prime}$ already reached its maximum. The maximum of $\operatorname{Im}(Y(\omega))$ and consequently the resistance per unit length, can be shifted beyond the FMR for a finite width microstrip. From (21), for the same value of $\mu_{\mathrm{e}}^{\prime \prime}$, the amplitude of $\operatorname{Im}(Y)$ is higher, if $\mu_{e}^{\prime}$ is negative, which explains the enhancement of the ferromagnetic absorption above the FMR. The presence of dielectric layers will not change the picture.

We next turn to the effect of the conductivity of the ferromagnetic film on the microstrip performance. Given the conductivity of $\mathrm{Ni}_{80} \mathrm{Fe}_{20}\left(6.25 \times 10^{6} \mathrm{~S} / \mathrm{m}\right)$ and the thickness $a=0.25 \mu \mathrm{m}$, the condition $2 k_{F} a<<1$ doesn't hold at frequencies above $0.1 \mathrm{GHz}$. This means that we can't use the approximation $\theta \equiv \tanh \left(2 k_{F} a\right) /\left(2 k_{F} a\right) \sim 1$ in Eq.(19). The quantity $\tilde{\mu}=\mu_{\mathrm{e}} \theta$, now replaces $\mu_{\mathrm{e}}$ in the equation for the nonconductive magnetic core. This quantity, in fact, represents the effective permeability in presence of eddy-currents in the ferromagnetic film ${ }^{14)}$. It leads to the slow drop of inductance at frequencies below $\omega_{R}$. This effect is stronger for thicker cores, or cores with higher conductivity.

\section{Conclusions}

The calculations describing the frequency behavior of the microstrip with a $\mathrm{NeFe}$ ferromagnetic core and two isolating dielectric layers, are provided. It is shown, that 
the slow drop of the inductance at frequencies below the FMR frequency $\omega_{R}$, is caused by the finite conductivity of the ferromagnetic core. The frequency $\omega_{\mathrm{RL}}$, at which the inductance of the microstrip vanishes, is higher than $\omega_{\mathrm{R}}$ for the microstrip with a finite width. The wavelength and attenuation as a function of frequency were calculated and demonstrated a good agreement with experiment with the maximal wave shortening taking place at the FMR frequency.

\section{Acknowledgments}

M.Vroubel and Y. Zhuang thank for the financial support by Foundation for Fundamental Research on Matter (FOM), the Netherlands

\section{References}

1) Y.Zhuang, M.Vroubel, B.Rejaei, and J.N. Burghartz: Tran Magn. Soc. Jpn., 2 (in the same issue) (2002).

2) S. Ikeda, T.Sato, A.Ohshiro, K. Yamasawa and T. Sakuma: IEEE Trans. Magn., 37, 2903 (2001).
3) N.Cramer, D.Lucid, D.K.Walker, R.E.Camley and Z.Celinski: IEEE Trans. Magn., 37, 2903 (2001).

4) R.J.Astalos and R.E.Camley: J. Appl. Phys., 83, 3744 (1998).

5) R.E. Camley and D.L. Mils: J. Appl. Phys. 82, 3058 (1997).

6) E .Salahun, P.Queffelec and G. Tanne: J. Appl. Phys., 91, 5449 (2002).

7) A.K. Bhattascharyya: Electromagnetic Fields in Multilayered Structures. Theory and Applications (Arthech House, 1994).

8) C. Nguyen: Analysis Methods for RF Microwave, and Milimeter-Wave Planar Transmission Line Structures. (Wiley-Interscience Publication, 2000).

9) A.G. Gurevich, G.A. Melkov: Magnetization, Oscillation and Waves (CRC Pres, 1996).

10) N.Bloembergen: Phys.Rev., 78, 572 (1950).

11) F.Bloch: Phys.Rev., 70, 460 (1946).

12) G.Perrin, J.C.Peuzin, O.Acher: J. Appl. Phys., 81, 5166 (1997).

13) A. Sustanskii, V.Korenivski, A. Gromov: J. Appl. Phys., 89, 775 (2001).

14) Y.Shimada, J.Numazawa, Y.Yoneda,A.Hosono: $J$ Magn. Soc. Jpn., 15, 327 (1991).

(Received June 7, 2002: Accepted October 18, 2002) 